\title{
Review: electroconvulsive therapy may be an effective short term treatment for people with depression
}

The UK ECT Group. Efficacy and safety of electroconvulsive therapy in depressive disorders: a systematic review and meta-analysis. Lancet 2003, Mar;361:799-808.

\section{QUESTION: What is the efficacy and safety of electroconvulsive therapy (ECT) for people with depressive illness?}

\section{Design}

Systematic review with meta-analysis.

\section{Data sources}

The reviewers searched Medline, Embase, the Cochrane Collaboration Depressive Anxiety and Neurosis and Schizophrenia Group Controlled Trial Registers, Cochrane Controlled Trials Register, Biological Abstracts, CINAHL, LILACS, PsycInfo, SIGLE, reference lists, and specialist textbooks. The full search strategy is available at http://image.thelancet.com/extras/ 02art8375webappendix.pdf.

\section{Study selection}

Unconfounded randomised controlled trials were eligible if they compared ECT with no ECT, ECT versus pharmacotherapy, or different forms of ECT for people with depressive illness. Non-randomised studies assessing mortality after ECT were also eligible, as were casecontrol neuroimaging and post-mortem studies examining structural brain changes following ECT. When available, evidence from randomised trials was included in preference to other designs.

\section{Data extraction}

Two reviewers selected papers for inclusion. Paired members of the review team extracted data independently. The authors assessed the quality of studies using methods appropriate to the specific design. For example, the quality of randomised trials was assessed via reporting of allocation concealment, blinding, loss to follow up, and length of follow up. Disagreements were resolved by discussion. The main outcome measures were depressive symptoms, immediate and long term effects on cognitive function, and mortality. The primary outcome for estimating efficacy was change in symptoms on a continuous depressive symptom scale at the conclusion of ECT. The reviewers undertook metaanalysis of data on short term efficacy.

\section{Main results}

73 trials met the inclusion criteria. ECT reduced depressive symptoms more effectively than pharmacotherapy (standardised effect size $-0.80,95 \%$ CI -1.29 to -0.29 , 1144 participants in 18 trials). There was no difference in depressive symptom outcomes when ECT was administered twice per week compared to three times per week, or once weekly versus three times per week. There was a trend towards greater cognitive impairment from more frequent ECT administration (210 participants in 6 trials). High doses of ECT were associated with greater reductions in depressive symptoms, but there was some evidence of greater cognitive impairments with high versus lower dose ECT (342 participants in 7 trials).

Real ECT reduced depressive symptoms more effectively than simulated ECT (standardised effect size $-0.91,95 \%$ CI -1.27 to $-0.54,256$ participants in 6 trials).
Bilateral ECT was more effective than unipolar ECT (standardised effect size $-0.32,95 \%$ CI -0.46 to -0.19 , 1408 participants in 22 trials).

\section{Conclusions}

Electroconvulsive therapy (ECT) appears to be an effective short term treatment for depression. It may be more effective than medication. High dose ECT appears more effective than low dose ECT for reducing depressive symptoms. Bilateral ECT appears moderately more effective than unilateral ECT.

\section{COMMENTARY}

Despite considerable progress in pharmacological treatments for depressive disorders, many patients only partially respond to antidepressant treatment. Resistance to antidepressants is a significant problem in clinical practice and ECT may offer some advantages. There is ongoing debate about the position of ECT in the armamentarium of biological treatments for depression. Many psychiatrists refuse to use it for ethical reasons. This excellent review uses sound methodology to summarise high quality evidence about the safety and efficacy of ECT for people with depressive disorders. The findings suggest that, despite ethical concerns, ECT is an effective short term treatment for depression and is likely more effective than drug therapy.

Unfortunately, the majority of studies comparing ECT with antidepressants focussed on tricyclics or irreversible MAO inhibitors. Only one study assessed the comparative efficacy of paroxetine versus ECT. Thus, the generalisability of these findings to SSRIs and other newer antidepressants used most frequently in psychiatric practice remains uncertain.

One disadvantage of ECT is that without follow up treatment its effects last for only a few months. The relapse rate without continuation treatment has been estimated to be between $50-95 \%$, with the majority of relapses occurring in the first 6 months. ECT is increasingly combined with antidepressants to improve acute phase response, although little data exists to support this practice.

Treatment resistance and limits in the effectiveness of pharmacologic treatments have led to renewed interest in ECT. Although this careful review provides information about many issues related to the efficacy and safety of different ECT modalities, a number of questions remain for clinicians and patients. These include: How frequently should ECT be administered in continuation and maintenance phase treatment? What are the long term adverse effects of maintenance phase treatment? Is combining ECT with pharmacotherapy superior to ECT or pharmacotherapy alone? Is ECT a safe procedure for outpatients? If we can answer these questions, ECT may become an increasingly important option for managing severe depression.

Michael Bauer, PhD MD Charité University Hospital Humboldt University at Berlin Berlin, Germany
UK Secretary of State for Health.

For correspondence: J Geddes, Department of Psychiatry, University of Oxford, Warneford Hospital, Oxford, UK. john.geddes@ psych.ox.ac.uk 